\title{
Cognitive Control of Saccadic Selection and Inhibition from within the Core Cortical Saccadic Network
}

\author{
Andreas Jarvstad ${ }^{1,2}$ and Iain D. Gilchrist ${ }^{1}$ \\ ${ }^{1}$ School of Experimental Psychology, University of Bristol, Bristol, BS8 1QU, United Kingdom, and 2Department of Experimental Psychology, University of Oxford, \\ Oxford, OX2 6GG, United Kingdom
}

The ability to select the task-relevant stimulus for a saccadic eye movement, while inhibiting saccades to task-irrelevant stimuli, is crucial for active vision. Here, we present a novel saccade-contingent behavioral paradigm and investigate the neural basis of the central cognitive functions underpinning such behavior, saccade selection, saccade inhibition, and saccadic choice, in female and male human participants. The paradigm allows for exceptionally well-matched contrasts, with task demands formalized with stochastic accumulation-to-threshold models. Using fMRI, we replicated the core cortical eye-movement network for saccade generation (frontal eye fields, posterior parietal cortex, and higher-level visual areas). However, in contrast to previously published tasks, saccadic selection and inhibition recruited only this core network. Brain-behavior analyses further showed that inhibition efficiency may be underpinned by white-matter integrity of tracts between key saccade-generating regions, and that inhibition efficiency is associated with right inferior frontal gyrus engagement, potentially implementing general-purpose inhibition. The core network, however, was insufficient for saccadic choice, which recruited anterior regions commonly attributed to saccadic action selection, including dorsolateral prefrontal cortex and anterior cingulate cortex. Jointly, the results indicate that extra-saccadic activity observed for free choice, and in previously published tasks probing saccadic control, is likely due to increased load on higher-level cognitive processes, and not saccadic selection per se, which is achieved within the canonical cortical eye movement network.

Key words: cognitive control; eye-tracking; fMRI; inhibition; saccades; selection

\section{Significance Statement}

The ability to selectively attend to, and to not attend to, parts of the world is crucial for successful action. Mapping the neural substrate of the key cognitive functions underlying such behavior, saccade selection and inhibition, is a challenge. Canonical tasks, often preceding the cognitive neuroscience revolution by decennia, were not designed to isolate single cognitive functions, and result in extremely widespread brain activity. We developed a novel behavioral paradigm, which demonstrates the following: (1) the cognitive control of saccades is achieved within key cortical saccadic brain regions; (2) individual variability in control efficiency is related to white-matter connectivity between the same regions; and (3) widespread activity in canonical tasks is likely related to higher-level cognitive demands and not saccadic control.

\section{Introduction}

When we interact with the world, visually salient, and less salient but nevertheless task-relevant objects, compete for attention. In the driving scene in Figure $1 A$, the most salient object is the road sign, yet the car on the left is the most relevant (will it pull out?). As in this example, there is often a tension between salience and

\footnotetext{
Received May 24, 2018; revised Sept. 10, 2018; accepted Dec. 11, 2018.

Author contributions: A.J. and I.D.G. edited the paper; A.J. and I.D.G. designed research; A.J. performed research; A.J. and I.D.G. contributed unpublished reagents/analytic tools; A.J. and I.D.G. analyzed data; A.J. and I.D.G. wrote the paper.

This work was supported by Engineering and Physical Sciences Research Council EP/1032622/1, Biotechnology and Biological Sciences Research Council Research Development Fellowship BB/H021574/1 to I.D.G., and British Academy Postdoctoral Fellowship D-MAD, PF150005 to A.J.

The authors declare no competing financial interests.

Correspondence should be addressed to Andreas Jarvstad at andreas.jarvstad@city.ac.uk.

https://doi.org/10.1523/JNEUROSCI.1419-18.2018

Copyright $\odot 2019$ the authors $\quad 0270-6474 / 19 / 392497-12 \$ 15.00 / 0$
}

task relevance: selection must overcome salient signals (look at sign) in favor of less salient but task-relevant ones (look at car).

Compared with the modest demands in the driving example, previously published tasks involve very strong control demands. For example, the anti-saccade task involves inhibiting the urge to look at a sudden-onset highly salient peripheral stimulus, on an otherwise empty screen, while making a saccade of the same amplitude to its mirror location (Hallett, 1978). Other tasks involve similarly strong demands (e.g., countermanding: Brown et al., 2008; search step: Thakkar et al., 2014; Xu et al., 2017).

Saccades, including saccades directed to singly presented stimuli (pro-saccades), engage a core eye-movement network, which includes posterior parietal cortex (PPC), frontal eye fields (FEFs), supplementary eye fields (SEFs), basal ganglia, brainstem (e.g., superior colliculus), and cerebellum (for review, see Sparks, 2002; Munoz and Everling, 2004; McDowell et al., 2008; Müri and Nyffeler, 2008; Liversedge et al., 2011; Schall, 2015). The 
A

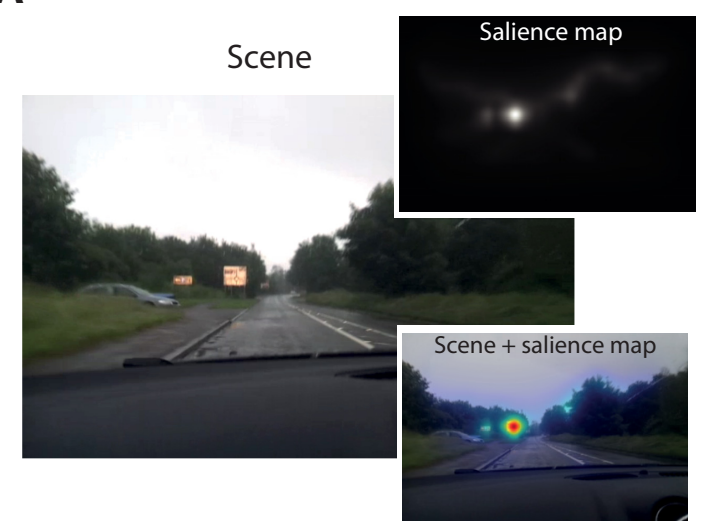

B

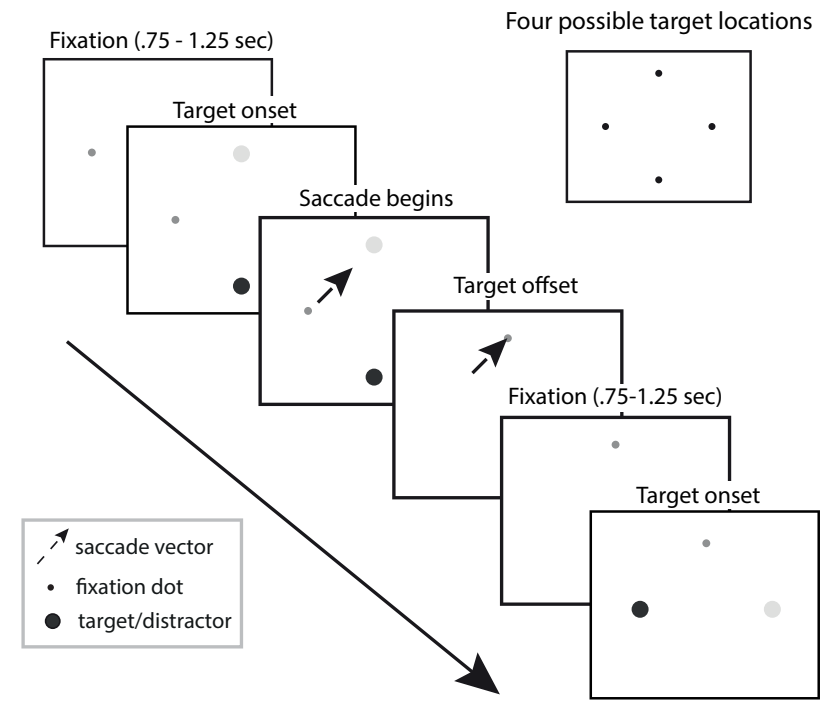

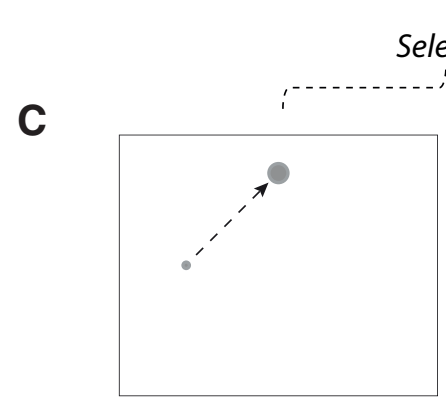

Pro-saccade

D

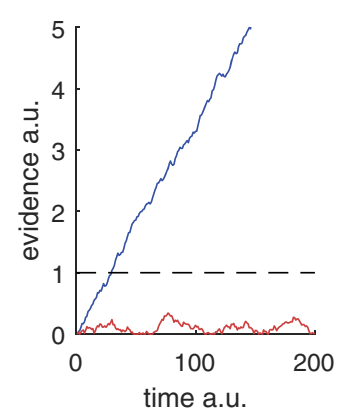

Selection

Inhibition
Choice

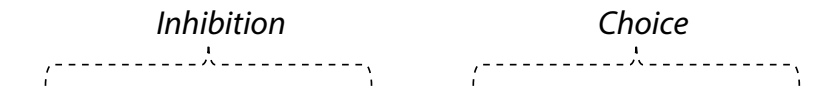

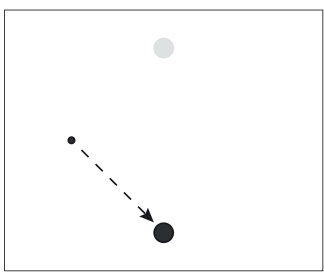

High-contrast

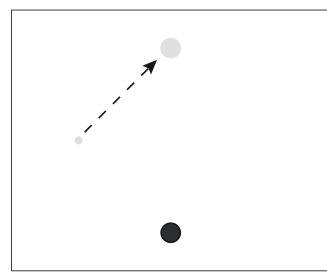

Low-contrast

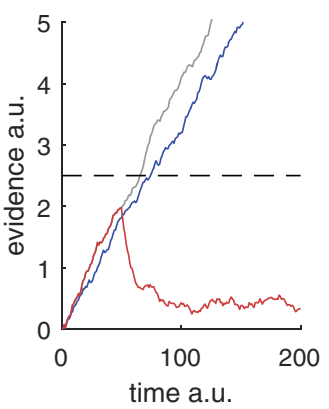

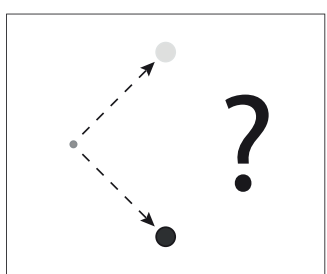

Choice
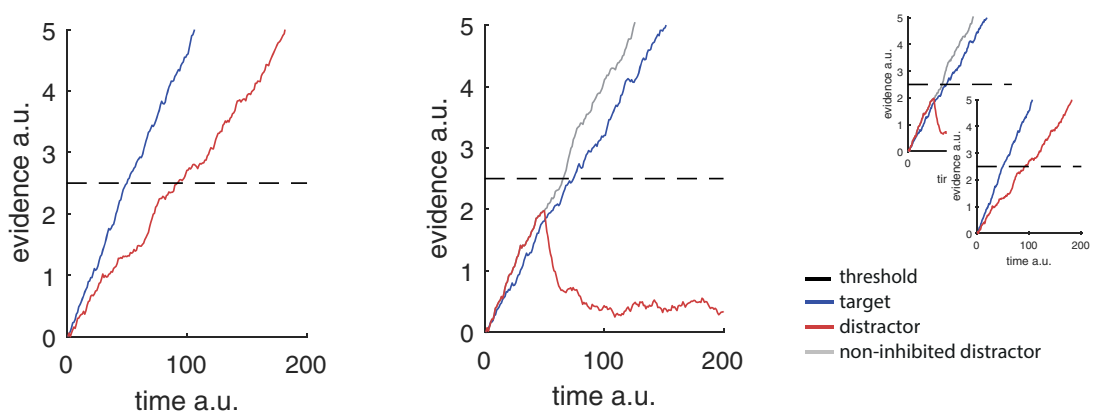

Figure 1. A, A driving scene, low-level visual salience map (Harel et al., 2006), and salience map overlaid on scene. The most salient part of the image is the road sign. The task-relevant car on the left has very low salience. $\boldsymbol{B}$, Trial structure. Each block of saccades began with fixation at one of the four possible target locations. After an interstimulus interval in the $0.75-1.25 \mathrm{~s}$ range $(\mathrm{sh}$ ifted and truncated exponential distribution), target stimuli were displayed. Once the eyes deviated by $>3^{\circ}$ from the current fixation, the targets were extinguished and replaced by a fixation dot at the target location. Blocks of $20 \mathrm{~s}$ of saccades were interleaved with blocks of $20 \mathrm{~s}$ of continuous fixation. C, Conditions. Pro-saccades involve saccading to a single target, high-contrast involves saccading to the most salient of two targets, low-contrast involves saccading to the least salient of two targets, and choice involves a free choice between the targets. Pairwise contrasts reflect: selection, inhibition, and choice. $\boldsymbol{D}$, Stochastic accumulator model. Blue traces represent the target accumulator. Red traces represent the distractor. Dashed line indicates the threshold at which a decision is made. Gray line for the low-contrast condition indicates the distractor without inhibition. The model includes within- and across-trial Gaussian noise and lateral inhibition.

network largely overlaps with networks for visual attention (Corbetta and Shulman, 2002).

The core network appears insufficient for tasks with strong control demands (e.g., anti-saccades), and additional cortical regions are recruited, including dorsolateral prefrontal cortex (dlPFC), anterior cingulate cortex (ACC), presupplementary motor area (pre-SMA), and inferior frontal gyrus (IFG) (McDowell et al., 2008; Chikazoe, 2010; Everling and Johnston, 2013; Thakkar et al., 2014). The recruitment of regions beyond the core network is interpreted as being linked to increased action control demands. Yet, given the tasks used, it is difficult to map regions onto unique cognitive functions (see also Hampshire et al., 2010; Thakkar et al., 2014; Xu et al., 2017).

For example, anti-saccades involve both inhibition and saccade vector inversion (Ford et al., 2005). Designs to deal with this include putting hypothesized processes on hold (Curtis and Connolly, 2008; Ettinger et al., 2008) or interrupting them (Brown et al., 2008). However, changing the temporal structure of the task or response may alter the processes themselves (Mazer and Gallant, 2003). Trials can also be sorted by behavioral criteria. One might, for example, assume that differences between error-free and erroneous anti-saccades reflect inhibition. However, there is 
often no unique mapping of sorted trials onto single cognitive functions. For example, error-related activity may also reflect failure to switch to, or maintain, the correct task set.

Crucially, relative to the standard baseline task (pro-saccades), anti-saccades are dramatically more error prone and therefore likely engage higher-level functions, such as performance monitoring linked to ACC (Botvinick et al., 2001; Braver et al., 2001) but also to FEF (Teichert et al., 2014). Moreover, anti-saccades involve a complex task set and therefore increased memory load, both linked to dlPFC function (Curtis and D'Esposito, 2003; Everling and Johnston, 2013). Right IFG (rIFG) activation, primarily seen in stopsignal tasks (Aron et al., 2004; but see Chikazoe et al., 2007), can similarly be given an alternative interpretation (Koechlin et al., 2003; Sharp et al., 2010; Levy and Wagner, 2011; Schall and Godlove, 2012; $\mathrm{Xu}$ et al., 2017). One possibility is that the recruitment of areas outside the core saccade network reflects general higher-level demands, and not saccadic control per se.

Here we introduce a novel saccade-contingent paradigm, which in combination with modest demands, allows for closely matched contrasts. We outline the paradigm and formalize its control demands with a parsimonious accumulation-to-threshold model. In addition to measuring $\mathrm{fMRI} B O L D$ as a function of changing action control demands, we also provide more exploratory analyses of the relationship between brain structure (white-matter integrity) and brain function (fMRI BOLD) and stable individual differences in saccadic reaction time (SRT).

\section{Materials and Methods}

\section{Paradigm}

The paradigm replaces the stop-start nature of standard tasks with a continuous sequence of saccades along a diamond configuration (see also Pertzov et al., 2011). Each sequence begins at one of four positions (potential target locations) (Fig. 1B). After a brief interstimulus interval, one or two stimuli (depending on condition) appear adjacent to this fixation (target onset). After saccade initiation, the stimuli are extinguished, and a new fixation dot is shown in place of the current target. This occurs before the saccade reaches its destination (target offset, saccade-contingent display). Another interstimulus interval follows, after which new potential targets are displayed adjacent to the now current fixation (and so on). To maximize the ability to detect differences in neural substrate between conditions, we use a blocked design, with periods of $20 \mathrm{~s}$ of saccades followed by periods of $20 \mathrm{~s}$ of fixation, although the paradigm is compatible with event-related designs.

There were four different conditions (Fig. 1C), three of which included two potential targets. The conditions were designed to capture differences in action control demands while minimizing between-condition differences in higher-level demands. In part, this was achieved by keeping overall error rates low $(\sim 2.5 \%$, see Results), through careful selection of stimulus parameters, and a prescan behavioral session (see Materials and Methods).

In the high-contrast condition, saliency and task relevance overlap and participants saccade to the most salient stimulus, equivalent to looking at the road sign in the driving scene. The low-contrast condition involves looking at the least salient stimulus and is equivalent to looking at the car; saliency and task relevance are in conflict. The first (pro) and the last (choice) conditions are extreme endpoints of the action selection spectrum. Pro-saccades provide an eye-movement baseline with minimal control demands, and choice mimics saccadic selection under "normal" conditions, when saccadic selection is free and involves mixtures of target saliences.

In the two-target conditions, one target was high contrast and the other low contrast. In an independent pilot study, we verified that the contrast of the stimuli affected their salience. The pilot was like the main study, except that only a single target stimulus was shown on each trial. That is, participants either looked at a singly presented high-contrast stimulus or a singly presented low-contrast stimulus. Saccades to high- contrast targets were faster than saccades to low-contrast targets $\left(z_{(8)}=\right.$ $-3, p=0.0195$, median difference $=5 \mathrm{~ms}$, Wilcoxon signed-rank test), thus confirming stimulus contrast as a determinant of stimulus salience (see also Ludwig et al., 2004).

Action selection can be captured with stochastic accumulation-tothreshold models (e.g., Carpenter and Williams, 1995; Shadlen et al., 1996; Trappenberg et al., 2001; Ludwig et al., 2005; Bompas and Sumner, 2011; Schall and Godlove, 2012). These models share the following assumptions: (1) potential actions compete for selection; (2) the evidence in favor of each action accumulates over time; (3) the accumulation process stops as soon as the evidence for one action reaches a decision threshold; and (4) the boundary-crossing action is selected for execution.

To formalize the demands associated with each condition (Fig. 1C), we used a parsimonious model, with minimal changes to account for differences between conditions. All conditions were modeled with two accumulators with deterministic starting points. Accumulator drift rates were proportional to the contrast of the stimulus (no distractor $<$ low $<$ pro $<$ high), and identical across conditions, subject to within- and across-trial Gaussian noise, and lateral inhibition and rectification (e.g., Usher and McClelland, 2001).

In the pro-saccade condition (Fig. 1C,D), there is only one target but two potential target locations. The target accumulator (blue line) is driven by a strong signal, and the distractor accumulator (red line) has close to zero-mean signal. Under these conditions, the threshold (dashed line) can be set very low: the absence of a distractor stimulus means that the risk of the wrong accumulator reaching threshold is negligible. The resulting saccades are both accurate and fast.

In the high-contrast condition (Fig. 1C,D), the task is to select the most salient stimulus (blue line). Unlike the pro-saccade condition, the distractor stimulus now has non-zero mean signal, which means that maintaining the same threshold would lead to frequent errors. The threshold is therefore elevated, which causes more evidence to be accumulated before action selection, thus avoiding premature selection of the less salient target. This, in turn, leads to longer response times with maintained accuracy.

Selecting the least salient target (Fig. $1 C, D$ ), however, cannot be achieved by threshold adjustment alone. On average, the low-contrast accumulator will not reach the threshold before the high-contrast accumulator. Thus, this condition necessitates a different kind of control. A mechanism that modulates the relative gain of the two accumulators, such that the weaker signal can overcome the stronger signal, allows the less salient stimulus to be selected. Here, this is achieved after an initial accumulation stage, after which the action for which there is more evidence is inhibited. It is these processes that are candidates for being implemented from outside the primary cortical saccade network.

Although the previous example relies on actively inhibiting the stronger signal, and the contrast is labeled inhibition, other mechanisms for modulating the relative strength of each accumulator, some of which do not (or do not only) rely on modulating the distractor accumulator, are also feasible. Additionally, inhibition is a multifaceted concept, both on the neural (e.g., lateral vs feedforward inhibition; different types of inhibitory interneurons) and the functional level (e.g., inhibition of prepotent responses, modulation of decision-relevant activity, global break-type inhibition, inhibition to stop persevering). All our conditions minimally involve some form of global break-type inhibition, to stop participants returning to neutral straight-ahead fixation (and/or move eyes freely). Importantly, only the low-contrast and the choice condition involve inhibition as in the anti-saccade and other tasks.

The fourth and final condition is choice. Unlike the high-contrast and low-contrast conditions, this task involves decisions about which of two decision rules to implement (possibly while considering the recent history of past choices). Participants were instructed not to preplan their saccades, or respond according to simple rules (e.g., alternating between low- and high-contrast stimuli), but to make a genuine choice on each trial. The main purpose of this instruction was to ensure that participants did not end up choosing only, or mostly, the high-contrast target. Because of the saccade-contingent display, any differences between this condition and the previous ones will be due to decision-related processes. 
As far as we are aware, there is no existing accumulator model for free saccadic choice. It is possible that participants simply switch randomly between selection and inhibition (Fig. $1 D)$. However, this model is likely too simplistic. Moreover, because errors are ill defined, the modeling of the cost of switching between mechanisms renders predictions very flexible; and because it is unclear how to model demands on performance monitoring and memory (e.g., remembering past choices), we refrain from specifying a full model for this condition.

The following contrasts between tasks may now be specified: pro-saccades contrasted against fixation quantifies the demands of making saccades. High-contrast - pro-saccades quantify additional demands, over and above those required for making saccades, imposed by selection, modeled as a threshold adjustment. Inhibition (low-contrast - high-contrast) reflects demands imposed by selection and additionally the control process, allowing the least salient signal to be selected. Choice (choice-low-contrast) represents the additional demands imposed by freely selecting between the two stimuli. Each consecutive contrast also captures the demands of the previous contrast(s). Inhibition, for example, also involves selection.

Based on extant literature, pro-saccades should engage the following cortical areas: PPC, FEF, and SEF, and visual cortex (McDowell et al., 2008). We expect demands associated with selection and inhibition to be met by the cortical saccadic network and higher-level visual areas (V4) (Mazer and Gallant, 2003). For the anti-saccade task specifically, it has been hypothesized that the increased fMRI BOLD in FEF (e.g., Curtis and D'Esposito, 2003) reflects inhibitory processes, possibly due to increased firing rates of fixation-holding neurons (Hanes et al., 1998), that the increased activity in PPC may reflect the vector inversion necessary for successful anti-saccades (e.g., Medendorp et al., 2005), and that SEF may contribute by slowing saccadic onsets (e.g., Boxer et al., 2006). Although our tasks do not require remapping, the PPC is more generally thought to provide salience signals for action selection (Paré and Dorris, 2011), and is thus likely to be involved in the encoding and modulation of targetrelevant signals in our task.

As noted initially, other tasks, including the anti-saccade task, which target the same functions as the current tasks, also engage regions outside the primary cortical saccade network (e.g., dlPFC, ACC, IFG). The dominant view of dlPFC function is that it is directly involved in inhibiting saccades (e.g., McDowell et al., 2008; Müri and Nyffeler, 2008). However, more recently, Everling and Johnston (2013) proposed that the antisaccade deficits observed in patients with dlPFC lesions (e.g., PierrotDeseilligny et al., 1991) and the increased fMRI BOLD for anti-saccades in dIPFC (e.g., Ettinger et al., 2008) are more consistent with the dlPFC implementing task sets. If the classical view of dlPFC is correct, we should observe dIPFC activity for the inhibition contrast here. More generally, if any extra-saccadic region is directly involved in saccadic selection and/or inhibition, they should be recruited for the current tasks also. Conversely, if the involvement of these regions in published tasks is due to increased higher-level demands (such as maintaining task sets), we should not be able to detect effects in extra-saccadic regions.

For choice, one might predict activations that look like a mixture of selection and inhibition. However, because choice is involved, areas more typically involved in decision-making tasks, such as ACC and dlPFC, should be engaged (e.g., Hare et al., 2011). Significant effects in these extra-saccadic regions for this contrast, in conjunction with no detectable effects for the inhibition and selection contrasts, would strongly suggest that these extra-saccadic regions do not implement selection or inhibition per se, but reflect higher-level cognitive functions (e.g., error monitoring, memory; see also Discussion) (Xu et al., 2017).
B
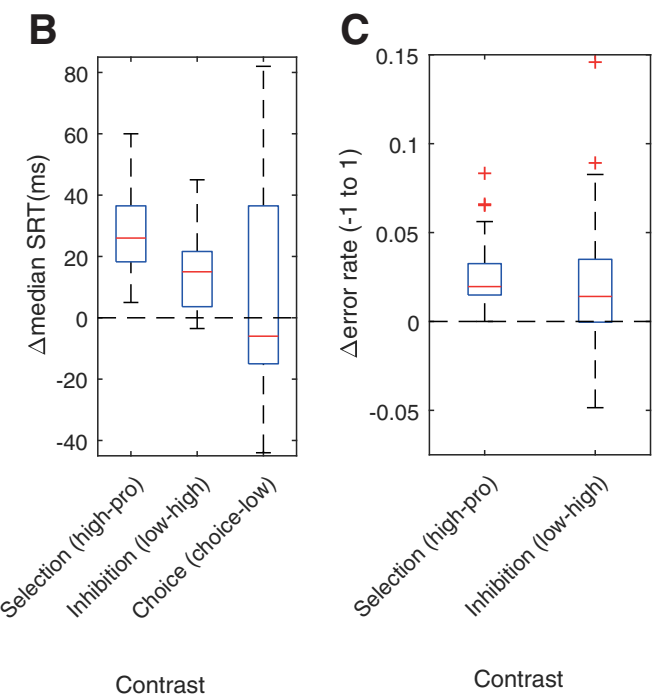

ondition

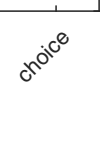

Contrast

Figure 2. fMRI session behavior. $A$, Median SRT as a function of condition. Error bars indicate IQR. $\boldsymbol{B}$, Boxplots of $\Delta S R T$ as a function of contrasts between pairwise conditions. $C$, Boxplots of differences in error rates ( $\Delta$ error rate) between pairwise conditions. Errors are undefined for the choice condition and therefore for the choice contrast.

\section{Procedure}

Participants took part in two sessions: a behavioral session $(\sim 60 \mathrm{~min})$ and a scanning session ( $\sim 90 \mathrm{~min})$. In both sessions, each participant performed two runs of the experimental tasks. In each run, participants performed six consecutive blocks of $20 \mathrm{~s}$ of fixation followed by $20 \mathrm{~s}$ of saccades, for each of the four conditions. Each six block sequence was preceded by a $5 \mathrm{~s}$ condition-cue. Condition order was randomized across runs and participants. For the three first conditions (Fig. 1), participants were instructed to look at the appropriate target. For choice, participants were told to make a genuine choice on each trial and to not preplan their eye movements. The behavioral session also included a familiarization phase to ensure compliance and understanding. The familiarization phase allowed participants to practice the tasks in a relaxed state, and its length was flexibly adjusted based on individual participant needs. Data from the familiarization phase were not recorded.

\section{Stimuli}

Targets were gray discs subtending $1^{\circ}$ visual angle presented on a light gray background $\left(346 \mathrm{~cd} / \mathrm{m}^{2}\right)$ at an eccentricity of 11.3 on the four cardinal axes ( $8^{\circ}$ from the center of the screen). The fixation dot subtended $0.15^{\circ}$. The high-contrast target was dark gray $\left(28 \mathrm{~cd} / \mathrm{m}^{2}\right)$, the lowcontrast target was light gray $\left(192 \mathrm{~cd} / \mathrm{m}^{2}\right)$, and the pro-saccade target was intermediate between the high and the low contrast target $\left(71 \mathrm{~cd} / \mathrm{m}^{2}\right)$. To reduce memory load, the fixation dot luminance matched the luminance of the current target, except in the choice condition where the luminance was intermediate between the two targets $\left(71 \mathrm{~cd} / \mathrm{m}^{2}\right)$.

\section{Apparatus}

The experiment was written in MATLAB using Psychtoolbox (Kleiner et al., 2007). In the behavioral session, the stimuli were shown on a CRT monitor $(1152 \times 864$ at $85 \mathrm{~Hz})$. In the fMRI session, the stimuli were back-projected using a DLP projector (F22 SX+ VisStim, ProjectorDesign $)$ onto a custom screen $(1400 \times 900$ at $60 \mathrm{~Hz})$ and viewed through a front-silvered head coil-mounted mirror. Eye movements in both the behavioral and fMRI sessions were recorded at $1000 \mathrm{~Hz}$ with an EyeLink 1000 (SR Research).

\section{Image acquisition}

Magnetic resonance images were acquired with a 32 channel head coil on a Siemens Skyra 3T scanner. For each participant, we recorded functional data, anatomical data, field maps and diffusion tensor images. EPIs were acquired with the following parameters: $\mathrm{FOV}=192 \mathrm{~mm}, \mathrm{TR}=2000 \mathrm{~ms}$, $\mathrm{TE}=30 \mathrm{~ms}$, flip angle $=90^{\circ}, 3 \mathrm{~mm}$ isotropic voxels with a $25 \%$ distance factor. Each volume consisted of 36 slices. T1 anatomical scans were 
acquired with the following parameters: $\mathrm{FOV}=240 \mathrm{~mm}, \mathrm{TR}=1800 \mathrm{~ms}$, $\mathrm{TE}=2.25 \mathrm{~ms}$, flip angle $=9^{\circ}$. Forty-nine field map slices were recorded with the following parameters: $\mathrm{FOV}=192 \mathrm{~mm}$, flip angle $=60^{\circ}$, $\mathrm{TR}=$ $520 \mathrm{~ms}, \mathrm{TE}=4.92 \mathrm{~ms}$. For each EPI sequence, we also recorded physiological variables (cardiac and respiratory phase). A total of 130 diffusion volumes, one without diffusion weighting, were acquired with Siemens Multi Directional Diffusion Weighting with an acceleration factor of 2, 2 $\mathrm{mm}$ slice thickness and a $30 \%$ distance factor, b value $=1400 \mathrm{~s} / \mathrm{mm}, \mathrm{FOV}$ $192 \times 192 \times 130 \mathrm{~mm}, \mathrm{TR}=6500 \mathrm{~ms}, \mathrm{TE}=70 \mathrm{~ms}$.

\section{Experimental design and statistical analyses}

The study was approved by the local ethics board, and participants gave written consent. Twenty-four healthy human participants of both sexes

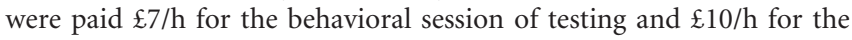
fMRI session (except for the first author [A.J.] who also took part). The sample size is consistent with the standard sample size for a study of this kind at the time of data collection. One of the participants was excluded as an outlier: in three of four conditions, this participant's median SRT was $>3$ interquartile range (IQR) relative to the other participants. This was a within-subject design, with each participant taking part in all four conditions: pro-saccade, high-contrast, low-contrast, and choice (Fig. 1). The study was not preregistered.

Behavioral analyses. Saccades, fixations, and blinks were extracted using SR Research's algorithms. Valid trials were defined as trials, which involved only one large saccade ( $>50 \%$ of the distance between fixation and target, or $>5.5^{\circ}$ ), for which the saccade start coordinate was within $3^{\circ}$ of the fixation dot and for which the saccade end coordinate was within $4^{\circ}$ of the target. The mean \pm SD of the percentage of trials classified as invalid were as follows: pro-saccade, $10 \pm 8 \%$; high-contrast, $8 \pm 5 \%$, low-contrast, $9 \pm 5 \%$; choice, $13 \pm 9 \%$. Saccade errors were defined as saccades that fulfilled the criteria for valid saccades but were directed at the wrong target (landing within $4^{\circ}$ of the nontarget stimulus). Errors are undefined in the choice task.

The tasks in the fMRI and behavioral sessions were identical, with periods of $20 \mathrm{~s}$ of saccades followed by periods of $20 \mathrm{~s}$ of fixation (see Procedure). Thus, time-on-task was time-limited, with fast participants completing, on average, more trials than slow participants. To account for this variation, regressors capturing saccade frequency and saccade errors were included in the GLM for the fMRI analyses (see below). We observed no quantifiable improvements (in SRT or error frequency) during the behavioral session, and report data from this session only to evaluate the association between behavioral and fMRI performance.

For behavioral analyses of SRTs and saccadic errors, nonparametric tests of differences (paired Wilcoxon) and tests of association (Pearson's $r)$ were used. All tests used conventional thresholds $(p \leq 0.05)$. To explore participants' choice sequences, we tested whether sequences were different from random (runs test, MATLAB, Bonferroni-corrected, for number of runs). We also assessed whether the average autocorrelation coefficients of participants choice sequences differed from zero by onesample $t$ tests (uncorrected).

fMRI analyses. Imaging data were analyzed with FSL (version 5.06-1, FMRIB's software library; www.fMRIb.ox.ac.uk/fsl). EPI data were corrected for head movements (Jenkinson et al., 2002), nonbrain removal was performed with BET (Smith, 2002), and data were spatially smoothed using a $5 \mathrm{~mm}$ FWHM Gaussian kernel. Data were filtered with a high-pass filter $(1 / 100 \mathrm{~Hz})$ and prewhitened (Woolrich et al., 2001). EPI data were corrected for distortions using field maps (Jenkinson, 2004) and were registered to participant anatomical space (Jenkinson et al., 2002), which in turn was registered to MNI space (nonlinear registration) (Andersson et al., 2007).

Statistical inference on EPI data was performed with a GLM. The design matrix was constructed as follows. Four regressors, one for each condition, specified the saccade blocks (with fixation blocks as the implicit baseline). Two further regressors specified eye blinks and the instruction periods, respectively. These regressors were convolved with a double-gamma function and its temporal derivative. Estimated head movement regressors were also included, as were regressors that removed the influence of volumes estimated to contain large head movements. Fi-
Table 1. SRT in milliseconds as a function of session type and run number ${ }^{a}$

\begin{tabular}{llllll}
\hline & Run & Pro-saccades & High-contrast & Low-contrast & Choice \\
\hline Behavior & 1 & $188(48)$ & $221(35)$ & $251(50)$ & $257(80)$ \\
& 2 & $196(36)$ & $226(21)$ & $245(27)$ & $237(71)$ \\
fMRI & 1 & $192(29)$ & $226(35)$ & $238(36)$ & $253(69)$ \\
& 2 & $201(37)$ & $228(34)$ & $240(26)$ & $236(70)$ \\
\hline
\end{tabular}

${ }^{a}$ Data are median values (IQRs).

nally, voxelwise physiological regressors, modeling the measured cardiac and the breathing cycle, were included (Brooks et al., 2008).

First-level (each run), second-level (the average of two runs), and third-level (participant-level) modeling involved six contrasts: (1) the pro-saccade condition was contrasted with the implicit baseline, targeting regions that are more active for mixtures of saccades and fixations compared with fixation; $(2-4)$ conditions with increasing demands on action selection were contrasted with those with lower demands: selection (2) high-contrast - pro-saccades, inhibition, (3) low-contrast high-contrast, and choice, (4) choice-low-contrast. We also included two control contrasts: (5) block-wise errors (number of valid saccades directed at the wrong target) and (6) block-wise saccade frequency (number of valid saccades), each modeled by a single regressor parametrically modulated by error and saccade frequency respectively. All regressors were entered competitively.

Second-level effects were modeled as fixed effects, and third- level effects were modeled using FSL's FLAME $(1+2)$ and FSL's automatic outlier deweighting (Woolrich et al., 2004). Statistical maps were clustercorrected at $z=2.3$ and $p=0.05$, and cluster information is provided in the tables. Brain areas were labeled using FSL's Harvard-Oxford Cortical and Subcortical atlases, the Cerebellar Atlas in MNI152 (FNIRT), and the Juelich Histological Atlas. Areas not labeled in these atlases (e.g., the hypothesized human homolog of FEFs), were localized anatomically and verified with published coordinates.

We also tested how individual differences in saccadic reaction time $(\mathrm{SRT})$, and differences between pairwise contrasts $(\Delta \mathrm{SRT})$, relate to functional activity and white-matter structure. Given the relatively low sample size $(N=23)$, these analyses should be viewed as exploratory. For functional analyses, standardized SRTs/DSRTs were entered as thirdlevel covariates.

Voxelwise statistical analysis of the diffusion data were performed using Tract-Based Spatial Statistics (TBSS) (Smith et al., 2006). Fractional anisotropy (FA) images were created by fitting a tensor model to the raw diffusion data using FMRIB's Diffusion Toolbox (FDT), and then brain-extracted using FSL's Brain Extraction Tool (BET). All subjects' FA data were then aligned into a common space using the nonlinear registration tool FNIRT, which uses a b-spline representation of the registration warp field (Rueckert et al., 1999). The mean FA image was created and thinned to create a mean FA skeleton, which represents the centers of all tracts common to the group. Each subject's aligned FA data were then projected onto this skeleton, and the resulting data were fed into voxelwise cross-subject statistics. Participants were median-split into low/high SRT and $\Delta$ SRT groups. Nonparametric permutation testing with threshold-free clustering (Winkler et al., 2014) was used to test for differences between groups.

\section{Results}

\section{Behavior}

The demands of successive conditions were reflected behaviorally in the fMRI session (Fig. 2A). Both selection and inhibition were associated with increased SRT (Fig. $2 B$ ), as shown by highly significant paired Wilcoxon tests (selection: $Z_{(22)}=-4.2, p<$ 0.0001 ; inhibition: $\left.Z_{(22)}=-3.9, p=0.0001\right)$. That is, although there were moderately large individual differences (see IQRs, Fig. $2 A$ ), there was highly reliable slowing down as control demands increased. The choice condition, however, did not result in statistically detectable change relative to the low-contrast condition (choice, $Z_{(22)}=-0.183, p=0.855$ ). 
Consistent with the paradigm targeting more modest control demands, SRT differences between conditions were smaller than those reported in the literature. For example, the behavioral cost of inhibition was $\sim 11 \mathrm{~ms}$ (low-contrast high-contrast SRT), with the reported behavioral cost of anti-saccades 6-10 times larger (anti-pro SRTs, e.g., 60 ms: Brown et al., 2006; >100 ms: Curtis and Connolly, 2008). The overall very low error rate $(\sim 2.6 \%$, compare $\sim 10 \%$ in Brown et al., 2006) is also consistent with the paradigm being less demanding than other tasks. Nonetheless, small but significant increases in error rates (Fig. 2C) were observed for both the selection and inhibition contrasts $\left(Z_{(22)}=-4.11, p<\right.$ 0.0001 and $Z_{(22)}=-2.524, p=0.0116$, respectively).

In the choice task, participants were effectively instructed to randomize their response from one trial to the next (see Materials and Methods). The purpose of this instruction was to avoid strong biases in favor of the high-contrast stimulus. Even so, there was a weak trend toward choosing the high-contrast target more than the low-contrast target (proportion of high-contrast choices, mean $\pm \mathrm{SD}$, $0.52 \pm 0.077)$, but it failed to reach significance $\left(t_{(22)}=1.4, p=0.17\right.$, one-sample $t$ test against a choice proportion of 0.5 ). Jointly with the small increases in SRT with increasing demands, and very small increases in errors, the approximately equal choice proportion, shows that, although the high-contrast stimulus was more salient than the low-contrast stimulus (see Paradigm), its prepotency was comparatively weak (compare Brown et al., 2008; Curtis and Connolly, 2008).

Testing for systematicity in response sequences is nontrivial, partly because tests are imperfect, we have limited data, but also because there is no single a priori definition of systematic/random given our task. Nonetheless, in addition to evaluating the overall choice proportion, we also explored participants choice sequences in each run (the sequence of low- and high-contrast target decisions).

First, we performed runs tests, which test the null hypothesis that the sequence of low- and high-contrast target choices was random. The null hypothesis was rejected in 4 of 46 tests (23 participants, 2 runs) at $p<0.025$ (Bonferroni-corrected for runs). Thus, only 0.087 of the choice sequences was classed as nonrandom, which is close to the expected Type I error rate. Second, we computed the autocorrelation of the choice sequence with a lag up to 21 for each run and participant. One-sample $t$ tests on the autocorrelation coefficients showed that responses at Trials 3 and 4 were negatively correlated with responses at Trial 1, but only for the first run. However, these effects were comparatively weak (average autocorrelation coefficient: Trial 3, $r=$ -0.10 ; Trial 4, $r=-0.11$ ).

In summary, participants chose the low- and high-contrast target in approximately equal proportion and choice sequences were consistent with random choice, although there were weak temporal dependencies in the first run. A choice strategy based on randomly switching between the low- and the high-contrast choice rules might be expected to result in SRTs in between that of the low- and high-contrast conditions. This was not observed, suggesting that choice was not achieved by a simple mixing of selection mechanisms.

Not only were average SRTs similar across the sessions and the runs (Table 1), SRT was highly correlated both across conditions and the two sessions (behavior and fMRI). Participants who were slow in one condition were also slow in others (pro-high, $r_{(21)}=$ $0.83, p<0.0001$; high-low, $r_{(21)}=0.79, p<0.0001$; choice-high, $r_{(19)}=0.63, p=0.0025,2$ participants with exaggerated slowing for choice excluded for the choice-high correlation). Furthermore, SRTs in the behavioral and the imaging session were highly correlated (pro-saccade: $\mathrm{r}_{(20)}=0.89, p<0.0001$; high-contrast: $r_{(20)}=0.89, p<0.0001$; low-contrast: $r_{(20)}=0.88, p<0.0001$; choice: $r_{(20)}=0.47, p=0.024, N=22$ due to missing behavioral data for 1 participant). We explore the functional and structural correlates of these apparently stable individual differences below.

\section{Pro-saccade contrast}

As expected, pro-saccades (Fig. 3), implicitly contrasted with fixation, recruited key saccadic cortical regions: PPC, the hypothesized human homolog of the FEFs at the junction of the prefrontal sulcus and the precentral sulcus (Luna et al., 1998; Amiez et al., 2006), and SEF, as well as visual areas (for cluster details, see Table 2). Within the FEF cluster, we observed typical lateral and medial peaks of activity (e.g., Amiez et al., 2006; Ettinger et al., 2008).

The weak ventromedial prefrontal cortex (vmPFC) activation may be due to the relative demanding peripheral fixation baseline 
Table 2. Local maxima in clusters of positive brain activation ${ }^{a}$

\begin{tabular}{|c|c|c|c|c|c|c|}
\hline Contrast & Cluster size (voxels) & Peak Z & Peak $x(\mathrm{~mm})$ & Peak $y(\mathrm{~mm})$ & Peakz (mm) & Peak region (MNI) \\
\hline \multirow[t]{6}{*}{ Pro-saccades } & 15,674 & 6.35 & 4 & -72 & 14 & Occipital lobe (V1/V2) \\
\hline & 3196 & 4.77 & 8 & 2 & 58 & SEF \\
\hline & 1195 & 5.62 & 48 & -2 & 48 & FEF \\
\hline & 782 & 4.29 & 6 & 56 & -14 & vmPFC \\
\hline & 419 & 3.85 & -24 & -8 & 12 & Putamen \\
\hline & 360 & 4.09 & 62 & -52 & 14 & Parietal/temporal lobe \\
\hline \multirow[t]{6}{*}{ Selection } & 4507 & 7.09 & -20 & -70 & 50 & PPC \\
\hline & 1295 & 4.94 & 26 & -62 & 48 & PPC \\
\hline & 784 & 3.74 & 34 & -66 & -14 & Occipital lobe (V4) \\
\hline & 718 & 4.21 & -26 & -4 & 50 & FEF \\
\hline & 385 & 3.93 & -38 & 4 & 34 & Precentral gyrus/MFG/BA44 \\
\hline & 289 & 4.2 & 28 & -2 & 52 & FEF \\
\hline \multirow[t]{4}{*}{ Inhibition } & 6537 & 5.68 & -18 & -56 & 56 & PPC \\
\hline & 552 & 4 & -20 & 2 & 56 & FEF \\
\hline & 347 & 3.74 & 20 & -86 & -6 & Occipital lobe (V2/V3) \\
\hline & 285 & 3.57 & 24 & 4 & 48 & FEF \\
\hline \multirow[t]{6}{*}{ Choice } & 21,133 & 7.87 & 36 & 44 & 36 & dIPFC (including FEF, SEF, ACC, IFG) \\
\hline & 9172 & 6.36 & -28 & -54 & 44 & PPC \\
\hline & 1830 & 5.92 & -34 & -58 & -32 & Cerebellum (crus I) \\
\hline & 1406 & 4.58 & 36 & -48 & -32 & Cerebellum (VI) \\
\hline & 437 & 3.75 & -6 & -20 & -4 & Brainstem (thalamus/SC) \\
\hline & 358 & 4.46 & 22 & 56 & -4 & $\mathrm{OFC}$ \\
\hline
\end{tabular}

${ }^{a}$ Cluster-forming threshold $z=2.3$ and $p<0.05$ (corrected). SC, Superior colliculus.

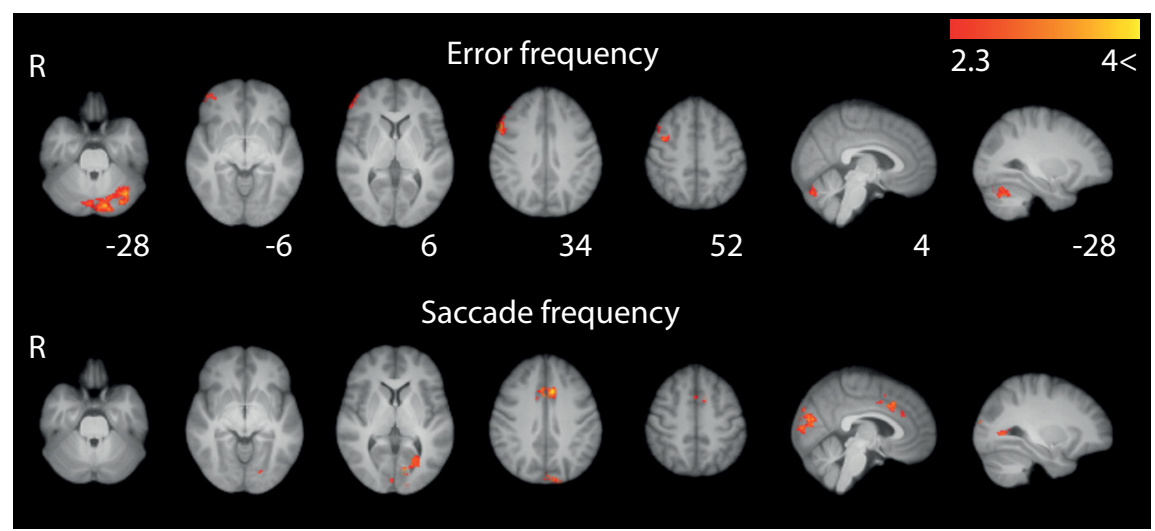

Figure 4. BOLD effects of error frequency and saccade frequency control regressors. Cooler colors represent greater activation. Maps were cluster-corrected at $z=2.3$ and $p=0.05$, and heat maps are scaled to lie in the $Z=2.3-4$ interval. Slice coordinates are in MNI space.

(deactivation of default network) (Raichle, 2015), which is consistent with greater vmPFC engagement for pro-saccades than for antisaccades (Pierce and McDowell, 2016). We also observed, within the left FEF cluster (Fig. 3; Table 2), a significant but relatively weak prefrontal activation. This activation was substantially more ventral and anterior than prefrontal activation commonly associated with higher-level control of saccades (Ettinger et al., 2008; e.g., Curtis and Connolly, 2008), and consistent with previously observed prefrontal pro-saccade activation (e.g., Brown et al., 2006).

All regressors were entered competitively, which explains the relative absence of subcortical, cerebellar, and brainstem activations. These regions become highly significant when control regressors are orthogonalized with respect to task regressors.

\section{Pairwise condition contrasts}

As can be seen in Figure 3, each additional demand, from an increase in decision threshold (selection), to a modulation of the integrated signals (inhibition), to free choice (choice), resulted in increased recruitment of key cortical saccade regions (PPC/FEF) and higher-level visual areas (see especially MNI $Z=52 \mathrm{~mm}$; Table 2 ).

The absence of significant activation outside the key cortical saccade regions for selection and inhibition suggests that action selection processes are implemented from within the cortical saccadic network, or at the very least, that action selection recruits saccadic regions to a much greater extent than extra-saccadic regions.

As initially noted, choice could be solved by mixing selection and inhibition mechanisms. However, the choice contrast evokes a much wider network than that one might expect by such mixing. Instead, many of the additional regions are also those extrasaccadic regions engaged by decisionmaking tasks (e.g., Hare et al., 2011), including dlPFC, ACC, SEF, pre-SMA, orbitofrontal cortex (OFC), and insula.

The observed main effects were not accounted for by differences in error rates or saccade frequency. Error frequency was associated with right lateral FEF extending down into right IFG/ OFC and a separate cluster in cerebellum (Fig. 4). Saccade frequency was associated with SEF/ACC (Talanow et al., 2016) and some posterior visual activity (Fig. 4). Both of these results were comparatively weak (peak $z=4.04$ in cerebellum for error and peak $z=3.89$ for frequency, compared with, e.g., peak prosaccade $z=5.72$ ).

\section{Neural correlates of individual $\Delta S R T$}

We also investigated how individual $\Delta$ SRT relate to BOLD (Fig. 5; Table 3). Effects of basic pro-saccade SRT were largely absent. The apparent lack of a significant association may be due to total on-task duration being fixed. This meant that faster participants completed more trials than slower participants, possibly masking BOLD-related efficiency differences (compare Ozyurt and Greenlee, 2011). 
Nevertheless, pairwise task contrasts revealed associations between the behavioral cost of action selection (i.e., $\Delta$ SRT between conditions, $\Delta$ SRT) and BOLD. Greater saccadic slowing for selection was associated with greater left dorsal IFG, PPC, and OFC activity (Fig. 5, red heat map). Faster participants (blue heat map), on the other hand, engaged vmPFC more, suggesting a greater investment in the task relative to fixation.

For inhibition and choice, those with larger behavioral costs showed increased activity in PPC and right insula/IFG. Thus, although our participants did not engage rIFG on average when having to inhibit a prepotent response (Fig. 3), there was nevertheless a relationship between the behavioral cost of inhibition and rIFG activity (Aron et al., 2004).

As can be seen in Figure 5, some whitematter voxels were significant. It is not unusual to observe significant BOLD responses outside the brain and/or in white matter in published work. Such activation may be a result of smoothing or imperfect mapping of EPI data onto the MNI template. To establish the extent of whitematter activations for these contrasts, we visually inspected the whole volume of each statistical map and ran FSLs atlasquery on the Harvard Oxford Subcortical Structural Atlas. Although a proportion of the significant voxels lay in white matter, the majority of significant voxels (for all contrasts) were classed as gray matter.

\section{White-matter integrity and SRT}

We further performed whole-brain TBSS analyses exploring the relationship between SRT and FA. We observed no significant effects for selection or choice (data not shown). For prosaccades, FA was higher for white-matter tracts between PPC and FEF for slower participants. The effect was largely leftlateralized and extended dorsally to basal ganglia (including thalamus, pallidum, and putamen) and anteriorly toward OFC. For these analyses, participants were median-split in low/high SRT groups, but post hoc inspection reveals a nonlinear relationship between FA and pro-saccade SRT (Fig. 6; Table 3), with a positive association between FA and SRT emerging for participants with pro-saccade SRT $\sim>190 \mathrm{~ms}$.

For inhibition, the effects were predominantly in the brainstem extending dorsally through thalamus and toward FEF. Specifically, participants with the smallest difference between low- and high-contrast SRT; that is, those with the smallest behavioral cost of inhibition had higher FA between cortical and subcortical eye movement regions. The relationship between mean FA and median SRT appears nonlinear here also, with the association levelling off for participants who pay the largest behavioral cost (largest $\Delta$ SRT).

\section{Discussion}

Salient stimuli have the capacity to drive saccadic selection; however, salient stimuli are not always task-relevant. When relevance and salience do not overlap, action selection mechanisms must overcome salient signals to select appropriate actions. Previous work has used tasks with very strong control demands, often capturing multiple cognitive functions. Here we introduced a novel paradigm involving more moderate demands allowing for well-matched contrasts.

While replicating key results, we also observe striking differences. The baseline condition (pro-saccades) engaged known cortical substrate for controlling eye movements. Compared with tasks that involve more extreme control demands, such as the anti-saccade task, however, both the selection and inhibition contrasts engaged a much smaller network, including PPC, FEF, and higher-level visual areas. These results suggest that cortical mechanisms for selection and inhibition are implemented within sensorimotor and integration regions, without strong reliance on more anterior regions.

The focal recruitment of PPC and FEF for selection and inhibition contrasts with the regions engaged when participants freely choose between targets, for which activations extend far beyond the core cortical saccade network (including dlPFC, ACC, insula, pre-SMA, SEF, and IFG). Although choice effectively involved switching between two task sets, the recruited networks are more extensive than those seen for task switching, or switching between trial types (Pierce and McDowell, 2017), and closely match those recruited for decision-making (e.g., Hare et al., 2011) (Fig. 3). Importantly, choice-related activations show that the absence of extra-saccadic cortical involvement for the preceding contrasts (selection, inhibition) was not due to a general inability to detect activity in these regions. 
Table 3. Positive BOLD $\times$ SRT and FA $\times$ SRT cluster details ${ }^{a}$

\begin{tabular}{|c|c|c|c|c|c|c|c|}
\hline & Size (voxels) & Peak $p$ & Peak Z & Peak $x(\mathrm{~mm})$ & Peaky $(\mathrm{mm})$ & Peakz (mm) & Peak region (MNI) \\
\hline \multirow[t]{4}{*}{$\mathrm{dSRT} \times$ selection } & 409 & - & 3.58 & -54 & -54 & -8 & Middle temporal gyrus \\
\hline & 332 & - & 3.85 & -30 & -70 & 58 & PPC \\
\hline & 330 & - & 3.76 & -42 & 8 & 28 & Left IFG, pars opercularis \\
\hline & 321 & - & 3.28 & -50 & 44 & -10 & Left frontal pole \\
\hline \multirow[t]{3}{*}{ dSRT $\times$ inhibition } & 651 & - & 3.89 & -28 & -72 & 28 & PPC extending to V4 \\
\hline & 368 & - & 3.73 & 12 & -66 & 68 & PPC \\
\hline & 254 & - & 3.39 & 56 & 16 & 12 & Right IFG, pars opercularis \\
\hline \multirow[t]{2}{*}{ dSRT $\times$ choice } & 564 & - & 3.97 & 48 & 42 & -8 & Right frontal pole ext to rIFG \\
\hline & 436 & - & 3.89 & -44 & -86 & -6 & Lateral occipital cortex, inferior division \\
\hline \multirow[t]{2}{*}{$\mathrm{FA} \times$ pro-saccade $S R T$} & 4145 & 0.03 & 3.79 & -26 & -30 & 19 & Superior corona radiata $\mathrm{L}$ \\
\hline & 788 & 0.037 & 4.64 & 26 & -23 & 26 & Posterior corona radiata $R$ \\
\hline \multirow[t]{8}{*}{$\mathrm{FA} \times$ inhibition dSRT } & 2048 & 0.036 & 5.3 & -9 & -20 & 8 & Thalamus/cerebral peduncle \\
\hline & 540 & 0.044 & 3.9 & 35 & -29 & 36 & Superior longitudinal fasciculus \\
\hline & 282 & 0.044 & 4.04 & -27 & -20 & -7 & Fornix (cres)/stria terminalis \\
\hline & 139 & 0.046 & 4.23 & 30 & 13 & 14 & Anterior corona radiata \\
\hline & 114 & 0.046 & 5.47 & 31 & -2 & 17 & External capsule $\mathrm{R}$ \\
\hline & 74 & 0.048 & 3.42 & -25 & 23 & 13 & Anterior corona radiata $\mathrm{L}$ \\
\hline & 63 & 0.049 & 3.42 & -28 & -29 & 9 & Internal capsule L \\
\hline & 39 & 0.048 & 4.42 & -23 & 29 & 1 & Anterior corona radiata $\mathrm{L}$ \\
\hline
\end{tabular}

${ }^{a}$ Cluster-forming threshold $z=2.3$ and $p<0.05$ (corrected) for SRT $\times$ BOLD. Threshold free clustering permutation testing for SRT $\times$ FA at $p=0.05$ (corrected).

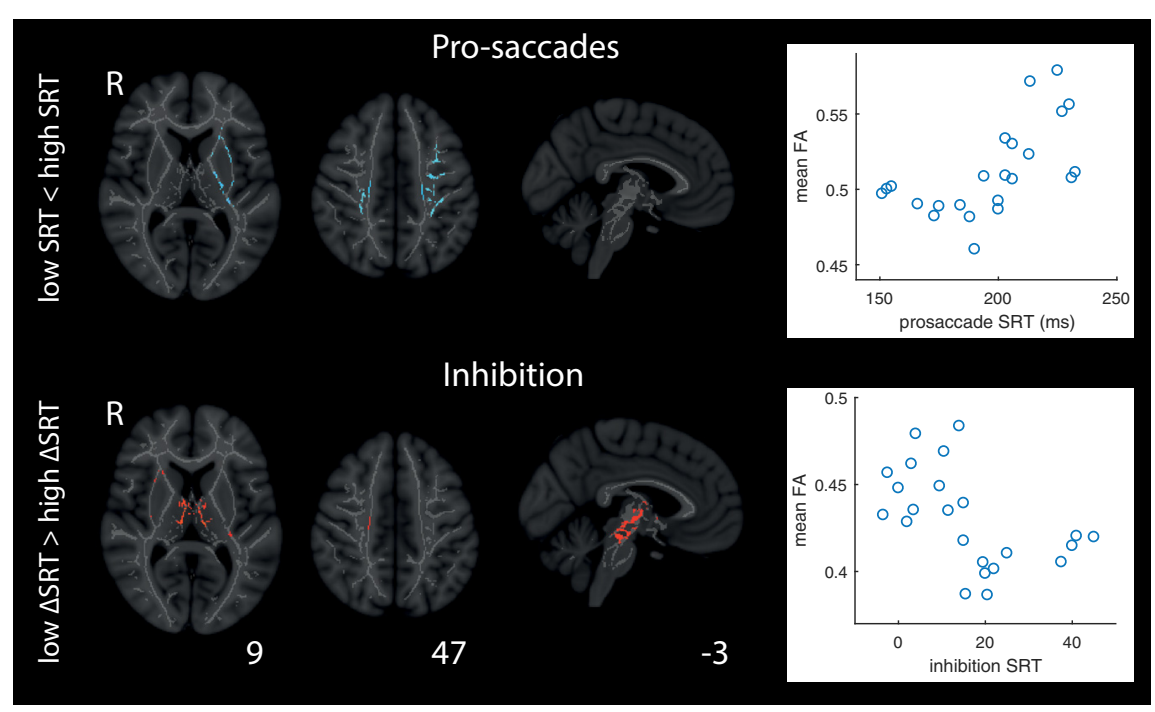

Figure 6. TBSS analyses of the relationship between SRT for pro-saccades, and $\Delta S R T$ for inhibition, and FA. Participants were median split into high and low SRT groups, and inferences performed on positive and negative associations between SRT and FA. Scatter plots represent the underlying continuous relationship between SRT (pro-saccades) and FA, and $\Delta S R T$ (inhibition) and FA averaged across all clusters of significant effects. Heat maps are probability maps thresholded at $p=0.05$ (corrected).

One explanation for the recruitment of extra-saccadic regions (e.g., dPFC, ACC) for previously published tasks, and their apparent lack of recruitment for selection and inhibition here, is that these regions are only engaged when control demands are more extreme. However, the joint result of no detectable SEF, ACC, dIPFC, or IFG engagement for the inhibition and selection contrasts, but very strong effects for the choice contrast, a condition with greater memory load and a more complex task set, is consistent with the hypothesis that effects in more typical tasks are due to higher-level processes, rather than saccadic action selection per se (see also Mostofsky et al., 2003; Everling and Johnston, 2013; Erika-Florence et al., 2014; Pierce and McDowell, 2016, 2017; Xu et al., 2017), which in turn is consistent with the reliable activation of these regions in tasks directly targeting these higher-level functions (e.g., Braver et al., 2001; Curtis and D'Esposito, 2003). This is especially noteworthy for dlPFC be- cause it is consistent with recent rethinking of dlPFC function, not as an inhibitory control region per se (Pierrot-Deseilligny et al., 1991; McDowell et al., 2008; Müri and Nyffeler, 2008), but as a region involved in maintaining and implementing task sets (Everling and Johnston, 2013; Johnston et al., 2014).

One possible exception is rIFG. Although the inhibition contrast did not result in significant rIFG engagement, the strength of rIFG activation correlated with $\Delta$ SRT for the two contrasts that do involve inhibition (inhibition, choice), but not significantly so for the contrasts that do not involve inhibition (prosaccades, selection). This is consistent with reported rIFG involvement in inhibition (e.g., Aron et al., 2004). However, the rIFG effect does not necessarily reflect a direct causal role in inhibition but may instead reflect broader inhibition-related demands (Erika-Florence et al., 2014; Xu et al., 2017). Post hoc tests showed a very strong relationship between $\Delta$ SRT cost for inhibition and pro-saccade SRT $\left(r_{(21)}=0.56, p=0.0059\right)$. That is, those who made fast pro-saccades also paid the highest cost for inhibition. rIFG activation may therefore reflect a compensatory mechanism in those who make fast pro-saccades, perhaps because they exhibit a lower baseline inhibition of all eye movement (e.g., less strong control of fixation).

Relatively little is known about the relationship between white-matter integrity and behavioral markers of saccadic control in humans (Manoach et al., 2007; Thakkar et al., 2016). We observed higher FA in white-matter tracts between PPC, FEF, and basal ganglia, for those who made slower pro-saccades. Higher FA is typically associated with improved task performance. However, higher FA has also been associated with poorer behavioral outcomes (Tuch et al., 2005; Hoeft et al., 2007) and neuropathology (Douaud et al., 2011). There are at least two possibilities for 
the current positive association: one related to what FA measures and the other related to the task itself. FA is affected by several properties of white-matter structure (Alexander et al., 2007), including myelination, fiber density, axonal diameter, and fiber crossings. Increased myelination leads to both higher FA and increased nerve conduction, the latter of which should improve SRT. However, as Tuch et al. (2005) outline, crossing fibers and other factors may invert the relationship, leading to a positive association.

Alternatively, slow pro-saccade SRT may be a sign of efficient task performance. All conditions involved relatively demanding fixation control. Stronger fixation holding is expected to result in slower release from fixation and therefore slower SRT. If stronger fixation is partly due to a taskwide strategy for avoiding premature responses, slower SRT should be correlated with performance. Post hoc analyses show that participants with slower pro-saccade SRT paid a smaller $\Delta$ SRT cost for selection $\left(r_{(21)}=\right.$ $-0.65, p<0.0001)$. Thus, slow pro-saccade SRT, associated with higher FA, may reflect more efficient fixation-holding mechanisms, allowing for better saccadic selection.

Moreover, participants who exhibited lower behavioral costs for inhibition had higher FA in tracts from the brainstem to FEF. These results are consistent with known white-matter tracts between saccade-generating regions in cortex, subcortex, and brainstem (e.g., Helminski and Segraves, 2003; for review, see Schall, 2015). A recent tractography study showed that a frontostriatal network is related to speed of inhibition and speed of selection in a search-step task (Thakkar et al., 2016). This is consistent with weak effects close to rIFG observed here, but direct comparisons are difficult because Thakkar et al. (2016) did not analyze whole-brain data and used a different task. Nevertheless, the observed effects suggest that individual differences in efficiency of inhibition may have structural origin.

Brain-behavior correlations are often explored in fMRI studies with sample sizes of $\sim 20$ participants. However, given recent problems in replicating brain-behavior correlations (Boekel et al., 2015), some caution is warranted in interpreting both the reported FAxSRT and the BOLDxSRT associations. Nonetheless, data on functional and structural origins of individual differences in saccadic action selection are sparse, and our results tentatively show that structural differences may underpin individual variability in action selection efficiency.

While the current paradigm achieves good mapping of hypothesized functions onto fMRI BOLD contrasts (inhibition and selection) (see also Xu et al., 2017), the accumulator formalization is likely too simplistic. The inhibition contrast, for example, was modeled as a modulation of the relative gain of two accumulators. However, the inhibition contrast strongly engaged FEF, PPC, and V4, and these regions perform overlapping but separable functions (Schall, 2015). Thus, a formalization, which more closely captures how the brain solves this task, will likely involve representing visual signals (V4), integrated decision variables (PPC), and motor plans (FEF) separately, all of which are modulated by the need to inhibit salient stimuli to select less salient but nevertheless task-relevant stimuli. At the level of individual neurons, we may speculate that the activity in some regions is supported by functional subclasses of neurons. Activity in FEF may, for example, be a result of an increase in the recruitment of fixation-holding neurons, but also a direct modulation of visuomotor neurons (Hanes et al., 1998).

Our paradigm could be used to explore distinct functions in different regions. One approach would be to use methods allow- ing for greater temporal specificity (MEG/neurophysiology), thus allowing the characterization of how signals in V4, PPC, and FEF evolve over the period of a single trial. Such approaches will necessarily involve event-related designs, which could also be used with fMRI to further probe processes involved in saccadic control. We also recognize that the choice contrast is not as optimal, in isolating function, as the other contrasts are. Of course, in some sense, the choice contrast provided the most interesting results. Nonetheless, future work is required to tease apart its widespread effects.

We presented a novel saccade-contingent paradigm for saccadic action selection under moderate demands, allowing for a very close mapping between hypothesized cognitive functions and fMRI BOLD contrasts. The results replicated the known network for simple eye movements (pro-saccades). However, selection and inhibition engaged a substantially more well-defined network than seen with alternative tasks. In contrast, when participants freely choose between targets, a substantially wider network is engaged: a network that overlaps with that seen in alternative tasks. Differences between the current and previous results can be accounted for, if it is assumed that extra-saccadic activity in standard paradigms is largely driven by higher-level demands associated with task complexity, not saccadic action selection per se. Finally, we observed associations between FA and behavior, which suggests that individual differences in saccadic action selection may be underpinned by individual differences in white-matter structure.

\section{References}

Alexander AL, Lee JE, Lazar M, Field AS (2007) Diffusion tensor imaging of the brain. Neurotherapeutics 4:316-329.

Amiez C, Kostopoulos P, Champod AS, Petrides M (2006) Local morphology predicts functional organization of the dorsal premotor region in the human brain. J Neurosci 26:2724-2731.

Andersson JL, Jenkinson M, Smith S (2007) Non-linear registration, aka Spatial normalisation (FMRIB technical report TR07JA2) pp 1-21. Oxford: University of Oxford FMRIB Analysis Group.

Aron AR, Robbins TW, Poldrack RA (2004) Inhibition and the right inferior frontal cortex. Trends Cogn Sci 8:170-177.

Boekel W, Wagenmakers EJ, Belay L, Verhagen J, Brown S, Forstmann BU (2015) A purely confirmatory replication study of structural brain-behavior correlations. Cortex 66:115-133.

Bompas A, Sumner P (2011) Saccadic inhibition reveals the timing of automatic and voluntary signals in the human brain. J Neurosci 31:1250112512.

Botvinick MM, Braver TS, Barch DM, Carter CS, Cohen JD (2001) Conflict monitoring and cognitive control. Psychol Rev 108:624-652.

Boxer AL, Garbutt S, Rankin KP, Hellmuth J, Neuhaus J, Miller BL, Lisberger SG (2006) Medial versus lateral frontal lobe contributions to voluntary saccade control as revealed by the study of patients with frontal lobe degeneration. J Neurosci 26:6354-6363.

Braver TS, Barch DM, Gray JR, Molfese DL, Snyder A (2001) Anterior cingulate cortex and response conflice: Effects for frequency, inhibition and errors. Cereb Cortex 11:825-836.

Brooks JC, Beckmann CF, Miller KL, Wise RG, Porro CA, Tracey I, Jenkinson M (2008) Physiological noise modelling for spinal functional magnetic resonance imaging studies. Neuroimage 39:680-692.

Brown MR, Goltz HC, Vilis T, Ford KA, Everling S (2006) Inhibition and generation of saccades: rapid event-related fMRI of prosaccades, antisaccades, and nogo trials. Neuroimage 33:644-659.

Brown MR, Vilis T, Everling S (2008) Isolation of saccade inhibition processes: rapid event-related fMRI of saccades and nogo trials. Neuroimage 39:793-804.

Carpenter RH, Williams ML (1995) Neural computation of log likelihood in the control of saccadic eye movements. Nature 377:59-62.

Chikazoe J (2010) Localizing performance of go/no-go tasks to prefrontal cortical subregions. Curr Opin Psychiatry 23:267-272.

Chikazoe J, Konishi S, Asari T, Jimura K, Miyashita Y (2007) Activation of right inferior frontal gyrus during response inhibition across response modalities. J Cogn Neurosci 19:69-80. 
Corbetta M, Shulman GL (2002) Control of goal-directed and stimulusdriven attention in the brain. Nat Rev Neurosci 3:201-215.

Curtis CE, Connolly JD (2008) Saccade preparation signals in the human frontal and parietal cortices. J Neurophysiol 99:133-145.

Curtis CE, D'Esposito M (2003) Success and failure suppressing reflexive behavior. J Cogn Neurosci 15:409-418.

Douaud G, Jbabdi S, Behrens TE, Menke RA, Gass A, Monsch AU, Rao A, Whitcher B, Kindlmann G, Matthews PM,Smith S (2011) DTI measures in crossing-fibre areas: increased diffusion anisotropy reveals early white matter alteration in MCI and mild Alzheimer's disease. Neuroimage 55:880-890.

Erika-Florence M, Leech R, Hampshire A (2014) A functional network perspective on response inhibition and attentional control. Nat Commun 5:4073.

Ettinger U, Ffytche DH, Kumari V, Kathmann N, Reuter B, Zelaya F, Williams SC (2008) Decomposing the neural correlates of antisaccade eye movements using event-related fMRI. Cereb Cortex 18:1148-1159.

Everling S, Johnston K (2013) Control of the superior colliculus by the lateral prefrontal cortex. Philos Trans R Soc Lond B Biol Sci 368:20130068.

Ford KA, Goltz HC, Brown MR, Everling S (2005) Neural processes associated with antisaccade task performance investigated with event-related fMRI. J Neurophysiol 94:429-440.

Hallett PE (1978) Primary and secondary saccades to goals defined by instructions. Vision Res 18:279-1296.

Hampshire A, Chamberlain SR, Monti MM, Duncan J, Owen AM (2010) The role of the right inferior frontal gyrus: inhibition and attentional control. Neuroimage 50:1313-1319.

Hanes DP, Patterson WF, Schall JD (1998) Role of frontal eye fields in countermanding saccades: visual, movement, and fixation activity. J Neurophysiol 79:817-834.

Hare TA, Schultz W, Camerer CF, O’Doherty JP, Rangel A (2011) Transformation of stimulus value signals into motor commands during simple choice. Proc Natl Acad Sci U S A 108:18120-18125.

Harel J, Koch C, Perona P (2006) Graph-based visual saliency. Proceedings of Neural Information Processing Systems 19:545-552.

Helminski JO, Segraves MA (2003) Macaque frontal eye field input to saccade-related neurons in the superior colliculus. J Neurophysiol 90: $1046-1062$.

Hoeft F, Barnea-Goraly N, Haas BW, Golarai G, Ng D, Mills D, Korenberg J, Bellugi U, Galaburda A, Reiss AL (2007) More is not always better: increased fractional anisotropy of superior longitudinal fasciculus associated with poor visuospatial abilities in Williams syndrome. J Neurosci 27:11960-11965.

Jenkinson M (2004) Improving the registration of B0-disorted EPI images using calculated cost function weights. Tenth International Conference on Functional Mapping of the Human Brain. Budapest, Hungary.

Jenkinson M, Bannister P, Brady M, Smith S (2002) Improved optimisation for the robust and accurate linear registration and motion correction of brain images. Neuroimage 17:825-841.

Johnston K, Koval MJ, Lomber SG, Everling S (2014) Macaque dorsolateral prefrontal cortex does not suppress saccade-related activity in the superior colliculus. Cereb Cortex 24:1373-1388.

Kleiner M, Brainard D, Pelli D (2007) What's new in Psychtoolbox-3? Perception 36, ECVP Abstr Suppl.

Koechlin E, Ody C, Kouneiher F (2003) The architecture of cognitive control in the human prefrontal cortex. Science 302:1181-1185.

Levy BJ, Wagner AD (2011) Cognitive control and right ventrolateral prefrontal cortex: reflexive reorienting, motor inhibition, and action updating. Ann N Y Acad Sci 1224:40-62.

Liversedge SP, Gilchrist ID, Everling S (2011) Oxford handbook on eye movements. Oxford: Oxford UP.

Ludwig CJ, Gilchrist ID, McSorley E (2004) The influence of spatial frequency and contrast on saccade latencies. Vision Res 44:2597-2604.

Ludwig CJ, Gilchrist ID, McSorley E (2005) The remote distractor effect in saccade programming: channel interactions and lateral inhibition. Vision Res 45:1177-1190.

Luna B, Thulborn KR, Strojwas MH, McCurtain BJ, Berman RA, Genovese CR, Sweeney JA (1998) Dorsal cortical regions subserving visually guided saccades in humans: an fMRI study. Cereb Cortex 8:40-47.

Manoach DS, Ketwaroo GA, Polli FE, Thakkar KN, Barton JJ, Goff DC, Fischl B, Vangel M, Tuch DS (2007) Reduced microstructural integrity of the white matter underlying anterior cingulate cortex is associated with increased saccadic latency in schizophrenia. Neuroimage 37:599-610.

Mazer JA, Gallant JL (2003) Goal-related activity in V4 during free viewing visual search: evidence for a ventral stream visual salience map. Neuron 40:1241-1250.

McDowell JE, Dyckman KA, Austin BP, Clementz BA (2008) Neurophysiology and neuroanatomy of reflexive and volitional saccades: evidence from studies of humans. Brain Cogn 68:255-270.

Medendorp WP, Goltz HC, Vilis T (2005) Remapping the remembered target location for anti-saccades in human posterior parietal cortex. J Neurophysiol 94:734-740.

Mostofsky SH, Schafer JG, Abrams MT, Goldberg MC, Flower AA, Boyce A, Pekar JJ (2003) fMRI evidence that the neural basis of response inhibition is task-dependent. Cogn Brain Res 17:419-430.

Munoz DP, Everling S (2004) Look away: the anti-saccade task and the voluntary control of eye movement. Nat Rev Neurosci 5:218-228.

Müri RM, Nyffeler T (2008) Neurophysiology and neuroanatomy of reflexive and volitional saccades as revealed by lesion studies with neurological patients and transcranial magnetic stimulation (TMS). Brain Cogn 68:284-292.

Ozyurt J, Greenlee MW (2011) Neural correlates of inter- and intraindividual saccadic reaction time differences in the gap/overlaps paradigm. J Neurophysiol 105:2438-2447.

Paré M, Dorris MC (2011) The role of posterior parietal cortex in the regulation of saccadic eye movements. Oxford handbook on eye movements. Oxford, UK:Oxford University Press.

Pertzov Y, Avidan G, Zohary E (2011) Multiple reference frames for saccadic planning in the human parietal cortex. J Neurosci 31:1059-1068.

Pierce JE, McDowell JE (2016) Modulation of cognitive control levels via manipulation of saccade trial-type probability assessed with event-related BOLD fMRI. J Neurophysiol 115:763-772.

Pierce JE, McDowell JE (2017) Contextual effects on cognitive control and BOLD activation in single versus mixed saccade tasks. Brain Cogn 115: 12-20.

Pierrot-Deseilligny C, Rivaud S, Gaymard B, Agid Y (1991) Cortical control of reflexive visually-guided saccades. Brain 114:1473-1485.

Raichle ME (2015) The brain's default mode network. Annu Rev Neurosci $38: 433-447$.

Rueckert D, Sonoda LI, Hayes C, Hill DL, Leach MO, Hawkes DJ (1999) Nonrigid registration using free-form deformations: application to breast MR images. IEEE Trans Med Imaging 18:712-721.

Schall JD (2015) Visuomotor functions in the frontal lobe. Annu Rev Vis Sci $1: 469-498$.

Schall JD, Godlove DC (2012) Current advances and pressing problems in studies of stopping. Curr Opin Neurobiol 22:1012-1021.

Shadlen MN, Britten KH, Newsome WT, Movshon JA (1996) A computational analysis of the relationship between neuronal and behavioural responses to visual motion. J Neurosci 16:1486-1510.

Sharp DJ, Bonnelle V, De Boissezon X, Beckmann CF, James SG, Patel MC, Mehta MA (2010) Distinct frontal systems for response inhibition, attentional capture, and error processing. Proc Natl Acad Sci U S A 107: $6106-6111$

Smith SM (2002) Fast robust automated brain extraction. Hum Brain Mapp 17:143-155.

Smith SM, Jenkinson M, Johansen-Berg H, Rueckert D, Nichols TE, Mackay CE, Watkins KE, Ciccarelli O, Cader MZ, Matthews PM,Behrens TE (2006) Tract-based spatial statistics: voxelwise analysis of multi-subject diffusion data. Neuroimage 31:1487-1505.

Sparks DL (2002) The brainstem control of saccadic eye movements. Nat Rev Neurosci 3:952-964.

Talanow T, Kasparbauer AM, Steffens M, Meyhöfer I, Weber B, Smyrnis N, Ettinger U (2016) Facing competition: neural mechanisms underlying parallel programming of antisaccades and prosaccades. Brain Cogn 107: $37-47$.

Teichert T, Yu D, Ferrera VP (2014) Performance monitoring in monkey frontal eye field. J Neurosci 34:1657-1671.

Thakkar KN, van den Heiligenberg FM, Kahn RS, Neggers SF (2014) Frontal-subcortical circuits involved in reactive control and monitoring of gaze. J Neurosci 34:8918-8929.

Thakkar KN, van den Heiligenberg FM, Kahn RS, Neggers SF (2016) Speed of saccade execution and inhibition associated with fractional anisotropy 
in distinct fronto-frontal and fronto-striatal white matter pathways. Hum Brain Mapp 37:2811-2822.

Trappenberg TP, Dorris MC, Munoz DP, Klein RM (2001) A model of saccade initiation based on the competitive integration of exogenous and endogenous signals in the superior colliculus. J Cogn Neurosci 13:256271.

Tuch DS, Salat DH, Wisco JJ, Zaleta AK, Hevelone ND, Rosas HD (2005) Choice reaction time performance correlates with diffusion anisotropy in white matter pathways supporting visuospatial attention. Proc Natl Acad Sci U S A 102:12212-12217.

Usher M, McClelland JL (2001) The time course of perceptual choice: the leaky, competing accumulator model. Psychol Rev 108:550-592.
Winkler AM, Ridgway GR, Webster MA, Smith SM, Nichols TE (2014) Permutation inference for the general linear model. Neuroimage 92:381-397.

Woolrich MW, Ripley BD, Brady M, Smith SM (2001) Temporal autocorrelation in univariate linear modelling of FMRI data. Neuroimage 14:1370-1386.

Woolrich MW, Behrens TE, Beckmann CF, Jenkinson M, Smith SM (2004) Multilevel linear modeling for fMRI group analysis using Bayesian inference. Neuroimage 21:1732-1747.

Xu KZ, Anderson BA, Emeric EE, Sali AW, Stuphorn V, Yantis S, Courtney SM (2017) Neural basis of cognitive control over movement inhibition: human fMRI and primate electrophysiology evidence. Neuron 96:14471458.e6. 